Preprint DFPD 94/TH/66, December 1994

\title{
DYNAMICS OF GENERALIZED COHERENT STATES
}

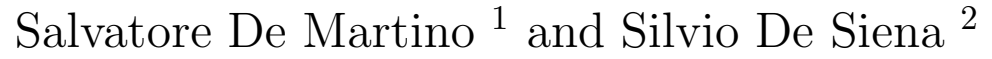 \\ Dipartimento di Fisica, Università di Salerno, \\ and INFN, Sezione di Napoli, Gruppo collegato di Salerno, \\ 84081 Baronissi, Italia \\ Fabrizio Illuminati คै \\ Dipartimento di Fisica "G. Galilei", Università di Padova, \\ and INFN, Sezione di Padova, \\ 35131 Padova, Italia
}

\begin{abstract}
We show that generalized coherent states follow Schrödinger dynamics in time-dependent potentials. The normalized wave-packets follow a classical evolution without spreading; in turn, the Schrödinger potential depends on the state through the classical trajectory. This feedback mechanism with continuous dynamical re-adjustement allows the packets to remain coherent indefinetely.
\end{abstract}

PACS numbers: 03.65.-w, 42.50.-p

\footnotetext{
${ }^{1}$ Electronic Mail: demartino@vaxsa.dia.unisa.it

${ }^{2}$ Electronic Mail: desiena@vaxsa.dia.unisa.it

${ }^{3}$ Electronic Mail: illuminati@mvxpd5.pd.infn.it
} 
Coherent states are by now of utmost importance in several areas of theoretical physics, ranging from quantum optics to statistical mechanics and quantum field theory [1]. They can be characterized as the quantum states that are closest to a classical, localized dynamics. The problem of defining and describing such states for general potentials traces back to Schrödinger, who in fact was able to give a complete, explicit solution only for the harmonic oscillator [2].

In modern language, as originally shown by Glauber [3], the harmonicoscillator coherent states are generated by the action of the displacement operator $D(\alpha)$ on the ground state. The same coherent states can also be described as eigenstates of the annihilation-operator or as the Heisenberg minimum-uncertainty states.

On the other hand, when one tries to generalize these three methods to general non-harmonic systems, different classes of generalized coherent states are obtained.

The displacement operator method, applied in the group-theoretical approach, yields the most satisfying characterization for it allows to preserve fundamental properties of the harmonic-oscillator coherent states such as overcompleteness and resolution of unity [4]; moreover, the displacement operator method can be applied without restriction to systems with general dynamical symmetry groups [由], [5]. In the following we will thus focus attention only on the generalized coherent states of the displacement operator, GCSs for brevity.

The major drawback of the group-theoretical description of GCSs is that in this approach it is very hard to understand the dynamical origin of their coherent behavior and to give an explicit description of their motion. For instance, one would like to know precisely in what sense they follow a classical evolution, i.e. the form of the potential appearing in the Schrödinger equation obeyed by a given GCS, the form of the potential appearing in the classical equation for the wave-packet center, and the relation among these two and the potential associated with the ground state being originally displaced by the action of $D(\alpha)$.

In this note we derive the explicit Schrödinger dynamics obeyed by GCSs. The main result of our analysis is that GCS are always associated to timedependent interactions, with a back-reaction mechanism between the state and the potential that is just what lets the packet remain indefinetely coherent without spreading. We sketch the proof in general and then discuss the 
explicit example of the Morse potential. Details of the general formalism and applications to other fundamental potentials will be presented elsewhere [6].

Our strategy is simply based on writing the displacement operator $D(\alpha)$ in the coordinate representation and then apply it to the ground state of general non-harmonic potentials. The dynamics is then reconstructed resorting to the Madelung hydrodynamic representation of Schrödinger equation.

This procedure yields non-stationary states whose wave-packet center exactly follows a classical equation of motion; they satisfy Schrödinger equation in a potential that is time-dependent through the trajectory solution of the classical equation for the wave-packet center. Thus the potential is subject to a back-reaction and keeps readjusting itself through the motion of the wave-packet center: this in turn allows the wave-packet to keep following the classical evolution without dispersion. In the limit of constant configurational expectation the time-dependent potential in the Schrödinger equation reduces to the potential whose ground state had been originally displaced.

Finally, the potential appearing in the classical equation for the wavepacket center is obtained as the time-independent part of the time-dependent potential in the given GCS. For sake of simplicity, in the following we discuss one-dimensional systems.

Consider, in the coordinate representation, the ground state $\Psi_{0}(x)$ of a time-independent configurational potential $V(x)$. Let the displacement operator $D(\alpha), \alpha(t)=\sqrt{2 \hbar}(Q(t)+i P(t))$, act on $\Psi_{0}(x)$; one obtains the non-stationary state $\Psi_{\alpha}(x, t)$ :

$$
\Psi_{\alpha}(x, t) \equiv D(\alpha) \Psi_{0}(x)=\exp \left(-i \frac{P Q}{2 \hbar}\right) \exp \left(i \frac{P}{\hbar} x\right) \Psi_{0}(x-Q),
$$

where $P(t)=\langle\hat{p}\rangle_{\alpha}$, expectation of the momentum operator in the state $\Psi_{\alpha}$, and $Q(t)=\langle\hat{q}\rangle_{\alpha}-\langle\hat{q}\rangle_{0}$, difference of the expectations of the coordinate operator in the states $\Psi_{\alpha}$ and $\Psi_{0}$. The ground-state mean $\langle\hat{q}\rangle_{0}=0$ for symmetric potentials; it is in general a non-zero constant for potentials with no definite parity.

The probability density $\rho_{\alpha}=\left|\Psi_{\alpha}\right|^{2}$ is a function of $\xi \equiv x-Q$, while the phase $S_{\alpha}$ is of the form $S_{\alpha}(x, t)=P x-P Q / 2$. In the hydrodynamic picture, to the complex Schrödinger equation for the state $\Psi$ there correspond two real coupled equations for the density $\rho$ and the phase $S$. We thus have the 
continuity equation

$$
\partial_{t} \rho=-\frac{1}{m}\left[\partial_{x}\left(\rho \partial_{x} S\right)\right]
$$

and the Hamilton-Jacobi-Madelung equation for the potential $V$ associated to Schrödinger dynamics:

$$
\partial_{t} S+\frac{\left(\partial_{x} S\right)^{2}}{2 m}-\frac{\hbar^{2}}{2 m} \frac{\partial_{x}^{2} \sqrt{\rho}}{\sqrt{\rho}}=-V .
$$

It is then easily shown by inserting $\rho_{\alpha}$ and $S_{\alpha}$ in the above equations that the GCS $\Psi_{\alpha}$ satisfies Schrödinger equation in the potential

$$
V(x, t)=\frac{\hbar^{2}}{2 m} F(\xi)-\frac{d P}{d t} x-\frac{P^{2}}{2 m}+\frac{1}{2}\left(\frac{d Q}{d t} P+\frac{d P}{d t} Q\right),
$$

where $F(\xi) \equiv\left(\rho_{\alpha}(\xi)\right)^{-1 / 2} d^{2}\left(\sqrt{\rho_{\alpha}(\xi)}\right) / d \xi^{2}$.

It follows from eq.(4) above that the potential depends on time through the expectations of the observables, which in turn are determined by the dynamical state of the system. Therefore we have proven that any GCS follows Schrödinger dynamics in a time-dependent potential. The latter has the same functional form of the original time-independent potential $V(x)$ associated to the ground state $\Psi_{0}$. The original dynamical system is recovered in the limit of constant classical solution $Q \rightarrow 0$.

One can also see that GCSs' wave-packet centers satisfy

$$
\frac{d P}{d t}=-\left.\partial_{x} V(x, t)\right|_{x=\langle\hat{q}\rangle}
$$

This is a coherence condition: the GCSs are driven by a classical evolution equation. The actual form of the classical equation can be read off expanding $V$ in powers of $x$ and equating coefficients of terms linear in $x$. This procedure allows to identify the potential $V_{\text {class }}(x)$ entering the classical equation as the time-independent part of the time-dependent potential entering Schrödinger equation. This back-reaction of the wave-packet motion on the Schrödinger potential is what allows the classical motion to hold exactly without dispersion. The price to be paid, with respect to the harmonic-oscillator case, is that in general $V_{\text {class }}(x)$ will not coincide with the original potential $V(x)$ associated to $\Psi_{0}$, unless the potential is symmetric. 
We will now elucidate the general structure outlined above by applying it to the solvable example of the Morse oscillator. Consider the potential $V(x)=U_{0}(1-\exp [-a x])^{2}$, where $U_{0}=\lambda^{2} \mathcal{E}_{0}$, and $\mathcal{E}_{0}=(\hbar a)^{2} / 2 m$. The depth of the different Morse wells is indexed by the adimensional constant $\lambda>1 / 2$. For computational convenience we choose the value $\lambda=1$. The corresponding ground state reads

$$
\Psi_{0}(x)=\left(\frac{2 \pi^{2}}{3 \Delta q^{2}}\right)^{\frac{1}{4}} \exp \left(-\gamma \frac{x}{\Delta q}-\exp \left(-2 \gamma \frac{x}{\Delta q}\right)\right)
$$

where the constant spreading $\Delta q^{2} \equiv\left\langle\hat{q}^{2}\right\rangle_{0}-\langle\hat{q}\rangle_{0}^{2}=4 \gamma^{2} / a^{2}$, and $\gamma=\pi / 2 \sqrt{6}$. Applying $D(\alpha)$ and solving the hydrodynamic equations for the potential yields

$$
V(x, t)=U_{0}(1-\exp [-a \xi])^{2}-\frac{d P}{d t} x-\frac{P^{2}}{2 m}+\frac{1}{2}\left(\frac{d Q}{d t} P+\frac{d P}{d t} Q\right) .
$$

From the above expression for the time-dependent potential one readily verifies that it reduces to the time-independent Morse well in the limit $Q \rightarrow 0$, and that condition (5) for classical motion is satisfied.

We must now identify the classical equation obeyed by $Q(t)$, that is the potential $V_{\text {class }}(Q)$. To this end one expands $V(x, t)$ in powers of $x$; identifying coefficients of the terms linear in $x$ one finally isolates the overall linear dependence $a(t) x$ in the potential $V(x, t)$ :

$$
a(t)=-\frac{d P}{d t}+2 a U_{0}(\exp (a Q)-\exp (2 a Q)) .
$$

Letting the arbitrary coefficient $a(t)=0$ the classical equation for $Q(t)$ reads

$$
\frac{d P}{d t}=2 a U_{0}(\exp (a Q)-\exp (2 a Q)) \equiv-\frac{d}{d Q} V_{\text {class }}(Q)
$$

so that the wave-packet center follows a classical motion in the potential

$$
V_{\text {class }}(Q)=U_{0}(1-\exp [a Q])^{2} .
$$

The above expression represents a repulsive Morse potential, obtained from the original one by letting $x \rightarrow-x$. This is not surprising, due to the non symmetric nature of the Morse oscillator. In fact, were we to define 
the displacement operator with the "wrong" sign, $\xi \rightarrow-\xi$, then $V_{\text {class }}(x)$ would coincide with the original Morse potential $V(x)$. On the other hand, $V_{\text {class }}$ exactly coincides with the original time-independent potential for symmetric systems, such as the harmonic oscillator with centripetal barrier, the symmetric Pöschl-Teller potential and the Coulomb potential.

Details on these systems and other results, including the description of generalized squeezed states and the study of time-evolution of GCSs with non-constant dispersion, will appear elsewhere [6].

We mentioned in the beginning that harmonic-oscillator coherent states are also states of minimum uncertainty. It is then possible along this line to define a different class of generalized coherent and squeezed states. This program was carried out by Nieto and co-workers [7], [8], who considered classically integrable systems allowing an energy-dependent canonical transformation such that the classical Hamiltonians are reduced, in the new "natural" variables, to quadratures. In this way one can quantize a system that is formally analogous to a harmonic-oscillator and seek the states that minimize the Heisenberg uncertainty product written in terms of the "natural" operators.

These minimum-uncertainty generalized coherent states present the interesting feature of satisfying an approximate classical motion for a finite time interval. In the light of our results this phenomenon finds a simple explanation: for non-harmonic systems a classical motion can be followed indefinetely only through the feedback mechanism driven by a time-dependent Hamiltonian. Since Nieto's coherent states are driven by a time-independent Hamiltonian, they eventually become delocalized after a finite time interval.

Harmonic-oscillator coherent states can also be described as the states that minimize the osmotic uncertainty product in Nelson stochastic quantization [9]. In the stochastic hydrodynamic picture they are states with classical current velocity (i.e. classical phase) and osmotic velocity linear in the process (i.e. Gaussian density) [10]. If one seeks for states still with classical phase but with densities not necessarily Gaussian, one recovers the displacement-operator generalized coherent states [11]. In fact, this result inspired us to study the dynamics of GCSs in the canonical picture.

In conclusion, we have presented the complete dynamical characterization of generalized coherent states, describing how to identify the classical equation for the coherent wave-packets and the back-reaction mechanism that is needed to preserve coherence in the time-evolution. 
In this sense generalized coherent states yield the first possible example of state-dependent interactions in nature. Beyond the conceptual importance in the field of quantum coherence, this result might be useful in applications.

For instance, in the study of particle beam dynamics, state-dependent Hamiltonians could be introduced to simulate the experimenter's "kicks" to keep the particle bundle coherent. In the case of time-dependent dispersion $\Delta q(t)$, these Hamiltonians could be of interest in the description of atomic wave-packets with large principal quantum numbers, the Rydberg states. 


\section{References}

[1] J.R. Klauder and B.S. Skagerstam, Coherent States (World Scientific, Singapore, 1985).

[2] E. Schrödinger, Naturwissenschaften 14, 664 (1926).

[3] R.J. Glauber, Phys. Rev. 130, 2529 (1963); Phys. Rev. 131, 2766 (1963).

[4] W.M. Zhang, D.H. Feng and R. Gilmore, Rev. of Mod. Phys. 62, 867 (1990).

[5] A.M. Perelomov, Generalized Coherent States and their Applications (Springer-Verlag, Berlin, 1986).

[6] S. De Martino, S. De Siena and F. Illuminati, to appear.

[7] M.M. Nieto and L.M. Simmons, Jr., Phys. Rev. Lett. 41, 207 (1978); M.M. Nieto, L.M. Simmons, Jr. and V.P. Gutschick, Phys. Rev. D23, 927 (1981).

[8] M.M. Nieto and D.R. Truax, Phys. Rev. Lett. 71, 2843 (1993).

[9] D. de Falco, S. De Martino and S. De Siena, Phys. Rev. Lett. 49, 181 (1982).

[10] S. De Martino, S. De Siena and F. Illuminati, Mod. Phys. Lett. B8, 977 (1994).

[11] S. De Martino, S. De Siena and F. Illuminati, Mod. Phys. Lett. B8, 1823 (1994). 